\title{
Sensitivity and Specificity of the Automated Squeeze Test (Gaenslen's Maneuver) For Identifying Metacarpophalangeal Synovitis By Magnetic Resonance Imaging
}

\author{
David Vega-Morales ${ }^{1 *}$, Jorge Antonio Esquivel ${ }^{1}$, Mario Alberto Garza-Elizondo ${ }^{1}$, María del Carmen \\ Larios-Forte ${ }^{1}$, Dionicio Ángel Galarza Delgado ${ }^{2}$, Lorena Pérez-Barbosa ${ }^{2}$ and Brenda Roxana \\ Vázquez-Fuentes ${ }^{1}$
}

${ }^{1}$ Department of Rheumatology Service and Internal Medicine, Hospital Universitario, México

${ }^{2}$ Department of Radiology, Hospital Regional Monterrey, Mexico

*Corresponding author: David Vega-Morales, Department of Rheumatology Service and Internal Medicine, Hospital Universitario, Mexico.

To Cite This Article: David VM, Jorge Antonio, Mario Alberto GE, María del Carmen LF, Dionicio Ángel GD, et al., Sensitivity and Specificity of the Automated Squeeze Test (Gaenslen's Maneuver) For Identifying Metacarpophalangeal Synovitis By Magnetic Resonance Imaging. 2020 - 8(5). AJBSR.MS.ID.001309. DOI: 10.34297/AJBSR.2020.08.001309.

Received: 眥 April 17, 2020; Published: 制 April 28, 2020

\section{Abstract}

Objective: To determine the diagnostic performance of the automated squeeze test (AST) on the metacarpophalangeal (MCP) joints to detect the presence of synovitis, edema or erosions by magnetic resonance imaging (MRI) by using the rheumatoid arthritis magnetic resonance imaging score (RAMRIS) in first-degree relatives (FDR) of Rheumatoid Arthritis (RA) patients with hand arthralgia and RA patients.

Methods: Observational and cross-sectional study for a diagnostic test that included 60 patients older than 18 years, divided into three groups: FDR with hand arthralgia group: 22 subjects; early RA group: 22 subjects who met ACR/EULAR 2010 Classification Criteria with less than two years since the onset of the symptoms; and late RA group: 16 patients who met ACR/EULAR 2010 Classification Criteria with more than two years since the onset of the symptoms. The AST was performed in the 60 participant's dominant hand and scored by RAMRIS.

Results: A total of 240 MCP joints were evaluated. The AUC for the total RAMRIS score $>10$ was [0.480 ( $95 \%$ CI 0.301-0.617) P=0.597]. For synovitis RAMRIS score $>7$ was $[0.459(95 \% \mathrm{CI} 0.331-0.669) \mathrm{P}=0.791]$ and for the presence of any synovitis by RAMRIS of [0.575 $(95 \% \mathrm{CI} 0.428-$ 0.723) $\mathrm{P}=0.331]$.

Conclusion: The AST maneuver positivity on the MCP's is not reliable to detect abnormalities on MRI.

Keywords: MRI; Squeeze Test; Arthritis; Diagnostic Performance

Abbreviations: AST: Automated Squeeze Test; MCP: Metacarpophalangeal; MRI: Magnetic Resonance Imaging; RAMRIS: Rheumatoid Arthritis Magnetic Resonance Imaging Score; FDR: First-Degree Relatives; RA: Rheumatoid Arthritis; ST: Squeeze Test; GCM: Gaenslen's Compression Maneuver; CSA: Clinical Suspect Arthralgia; RF: Rheumatoid Factor; ACPA's: Anti-Citrullinated Peptide Antibodies; ESR: Erythrocyte Sedimentation Rate; CRP: C-Reactive Protein; TR: Repetition Time; TE: Echo Time; NEX: Number of Excitations; FOV: Field of View; OMERACT: Outcome Measures in Rheumatoid Arthritis Clinical Trials; ROC: Receiving Operating Curve; AUC: Area Under the Curve; ICG: Indocyanine Green; FOI: Fluorescence Optical Imaging; OST: Optical Spectral Transmission; ULD-CT: Ultra-Low-Dose Computed Tomography

\section{Introduction}

Early identification and treatment of rheumatoid arthritis (RA) patients prevent joint destruction, deformity, and disability [1]. For this purpose, research to identify and early referral of individuals with arthralgia at risk of progression to RA has increased in the last years [2]. Even though there are many efforts to identify these subjects, it is fundamental to perform a clinical examination to determine the presence of synovitis and achieve an early referral 
to the rheumatologist. Studies have reported that only $20 \%$ of the general practitioners have recorded the realization of any clinical maneuver [3]. The squeeze test (ST) or Gaenslen's compression maneuver (GCM) positivity is characterized by tenderness upon lateral compression (squeezing) of the metacarpophalangeal (MCP) joints on a patient who has active synovitis $[4,5]$. The performance of this maneuver is included in several early referral strategies [6]. Recently, EULAR has established Clinical Suspect Arthralgia (CSA) as a definition, consisting in a set of seven parameters that describe patients with arthralgia with no definite diagnosis but a suspicion of progression to RA, within these parameters the positive ST is also included [7]. It has been demonstrated the presence of variability in the ST performance among rheumatologists, reporting that, in one second of compression, the force ranges between $0.400 \mathrm{~kg} / \mathrm{s} 2$ and $1.0 \mathrm{~kg}$, using the right hand and a superior approach on the MCP joints when performing the maneuver $[8,9]$. To solve these variabilities and discrepancies we have designed an automated compressor of MCP joints. And we have found that the mean force taken to evoke pain in patients with RA is 3.07 and $2.78 \mathrm{~kg}$ in right and left hand, respectively, in contrast to patients without arthritis who required 4.2 and $4.6 \mathrm{~kg}$ [10].

\section{Objective}

The aim of this study is to determine the diagnostic performance of the automated squeeze test (AST) on the MCP joints to detect the presence of synovitis, edema or erosions by magnetic resonance imaging (MRI) using the rheumatoid arthritis magnetic resonance imaging score (RAMRIS) [11-13] in first-degree relatives (FDR) of RA patients, of whom CSA in hands was suspected, as well as in RA patients.

\section{Materials and Methods}

\section{Study Design}

An observational and cross-sectional study for a diagnostic test was performed.

\section{Patients}

The inclusion criteria consisted in both sexes subjects older than 18 years-old, with RA diagnosis according to the ACR/EULAR 2010 classification criteria, with positive rheumatoid factor (RF) or anti-citrullinated peptide antibodies (ACPA's), recent ( $<1$ month) erythrocyte sedimentation rate (ESR) and C-reactive protein (CRP) results and serum creatinine test less than $1 \mathrm{mg} / \mathrm{dl}$, and FDR of RA patients with hand arthralgia who met at least 2 parameters of the CSA EULAR definition including being FDR of RA patients [7]. The participants were excluded if they had the following: pregnancy, lactation, chronic kidney disease, claustrophobia, allergies to the MRI contrast, deformities in hands that prevent staying 45 minutes in the resonator and/or individuals with prosthetic teeth, orthodontic apparatus or metallic devices that interfere with the MRI's action mechanism. Sixty consecutive subjects participated, and they were classified into three groups. Twenty-two were patients with hand arthralgia and were FDR of RA patients (CSA group), twenty-two with RA of less than 24 months since the onset of the symptoms (ERA group), and sixteen with established RA of more than 24 months since the onset of the symptoms (LRA group). All patients were recruited from the Rheumatology Service in a University Hospital in Monterrey, Mexico from July 2016 to January 2017. The Ethical Committee of the Autonomous University of Nuevo Leon approved the study protocol. All study participants have signed an informed consent with all the information about the protocol before the performance of any study.

\section{Automated Device}

The device consisted in an automated compressor, which is composed of two main elements: the lineal effector that provides the needed force and pressure to perform the maneuver and the 9 oppressor, which is a mechanical object that transmits the force from the lineal effector to the patient's hand [9]. In order to execute the ST, the device must be connected to a computer with the software Runtime LabVIEW 2013 NI-VISA 5.4 (Run-Time Support, Configuration Support) NI I/O Trace 3.1 NI Measurement \& Automation Explorer 5.5 NI System Configuration 5.5, which controls the compressor performance and stores the data. The subject's dominant hand is set in rest position with alignment of the fifth MCP joint and the oppressor is placed on the second MCP joint, subsequently, the application interface is used to control the initiation and ending of the compression. The device was used in the sixty participants and 3 measurements were taken. The compressions' mean force in which the patient expressed pain was recorded. The rheumatologist who performed the test did not have access to the patient's MRI results.

\section{Magnetic Resonance Imaging}

The gold standard study to evaluate the AST performance to detect synovitis was the MRI. MRI examined thedominant hand of the sixty participants from the three groups with Signa equipment Twin HDx1.5 Teslas of General Electric-GE with hand-wrist antenna and anti-inflammatory suspension, 24 hours prior to the study. To validate the presence of synovitis, all the MRI examinations were carried out with T1-weighted echo and T2-weighted fat suppression sequences with and without intravenous contrast. The T1 coronal scanning measures were as follows:

a) Repetition time (TR) $=600 \mathrm{msec}$, echo time (TE) $=10$ msec, Flip Angle = $137^{\circ}$;

b) Number of excitations $(\mathrm{NEX})=1$;

c) Field of view $(\mathrm{FOV})=192 \times 220 \mathrm{~mm}$;

d) Matrix $=450$ frequency, 310 phase; and

e) Slice Thickness $=2 \mathrm{~mm}$, interslice gap $=2 \mathrm{~mm}$. 
The T2 coronal scanning measures with fat suppression were as follows:
a) $\mathrm{TR}=3,010 \mathrm{msec}, \mathrm{TE}=55 \mathrm{msec}$, flip angle $=145^{\circ}$;
b) NEX = 1;
c) $\mathrm{FOV}=220 \times 220 \mathrm{~mm}$;
d) Matrix $=448$ frequency, 358 phase; and
e) Slice thickness $=2 \mathrm{~mm}$, Interslice Gap $=2 \mathrm{~mm}$.

The T1 axial scanning measures were as follows:
a) $\mathrm{TR}=640 \mathrm{msec}, \mathrm{TE}=13 \mathrm{msec}$, Flip Angle $=134^{\circ}$;
b) $\mathrm{NEX}=1$;
c) $\mathrm{FOV}=121 \times 130 \mathrm{~mm}$;
d) Matrix $=510$ frequency, 380 phase; and
e) Slice Thickness $=4 \mathrm{~mm}$, Interslice Gap $=6 \mathrm{~mm}$.

The T2 axial scanning measures were as follows:
a) $\mathrm{TR}=3,700 \mathrm{msec}, \mathrm{TE}=56 \mathrm{msec}$, Flip Angle $=150^{\circ}$;
b) NEX = 1;
c) $\mathrm{FOV}=121 \times 129 \mathrm{~mm}$;
d) Matrix $=380$ frequency, 250 phase; and
e) Slice thickness $=4 \mathrm{~mm}$, Interslice Gap $=6 \mathrm{~mm}[10]$.

The MRI findings were scored according to the OMERACT (Outcome Measures in Rheumatoid Arthritis Clinical Trials)Rheumatoid Arthritis Magnetic Resonance Imaging Score (RAMRIS) [12]. Two hundred and forty joints (MCP 2-5) were evaluated semi quantitatively (grades $0-3$ ) for synovitis, for bone erosion (0-10, increments of $10 \%$ according to the proportion of bone involved) and for bone edema $(0-3$, increments of $33 \%$ according to the proportion of bone involved). The radiologist did not have access to the patient's clinical and AST results.

\section{Statistical Analyses}

Descriptive statistics were used to analyze data. For the continuous variables, normality test was performed, and were described as mean or median and standard deviation or interquartile range, according to its Gaussian distribution. Categorical variables were expressed as frequency and percentages. Analytic statistics was performed to establish the difference between the synovitis, erosion and BME scores between groups. We explore three different MRI/RAMRIS MCP synovitis definitions:

a) Median RAMRIS synovitis score that statistically differentiate between CSA and RA (ERA and LRA) patients.

b) Median total RAMRIS score that differentiate between CSA and RA patients and

c) Presence or absence of synovitis by RAMRIS. Later, we evaluated the receiving operating curve (ROC) with the mean of the three AST forces on the dominant hand of each patient. The area under the curve (AUC) was calculated, as well as the sensitivity and specificity for each definition.

\section{Results}

The demographic and clinical data of the patients are reviewed in Table 1. A total of 240 joints of 60 consecutive patients were analyzed with the AST and MRI. We found a mean force to exert discomfort in MCF joints for $5.6 \mathrm{~kg} / \mathrm{s}^{2}$ (2.9 SD) in the whole cohort. Divided by group we found $5.2 \mathrm{~kg} / \mathrm{s}^{2}$ (3.1 SD), $6.9 \mathrm{~kg} / \mathrm{s}^{2}$ (2.9 SD) and $4.4 \mathrm{~kg} / \mathrm{s} 2$ (1.9 SD) in CSA, ERA and LRA respectively. We found statistical differences in the force between ERA and LRA ( $p=0.019)$, not for CSA vs ERA, nor CSA vs LRA. In Table 2, the RAMRIS scores are depicted divided by group. We found statistical differences between CSA vs ERA $(\mathrm{P}=0.035)$ and CSA vs LRA $(\mathrm{P}=0.022)$ in the synovitis RAMRIS score. We compared the erosion and BME scores between groups with no difference. The AUC for the total RAMRIS score $>10$ was [0.480 (95\% CI 0.301-0.617) $\mathrm{P}=0.597]$. For synovitis RAMRIS score $>7$ was [0.459 (95\% CI 0.331-0.669) $\mathrm{P}=0.791$ ] and for presence of any synovitis by RAMRIS was [0.575 (95\% CI 0.4280.723) $\mathrm{P}=0.331$ ]. For the synovitis RAMRIS score presence, the most sensitive and specific cut-off of the force by AST was $4.645 \mathrm{~kg} /$ $\mathrm{s}^{2}$ with a $66.7 \%$ sensitivity and $50 \%$ specificity. The most sensitive point was $1.29 \mathrm{~kg} / \mathrm{s}^{2}$ (95.8\% sensitivity) and the most specific was $10.3 \mathrm{~kg} / \mathrm{s}^{2}(93.7 \%)$.

\begin{tabular}{|c|c|c|c|c|}
\hline Table 1: Baseline characteristics. & \multicolumn{5}{|l|}{} \\
\hline & TOTAL & FDR n=22 & ERA n=22 & LRA n=16 \\
\hline Age Mean (SD) & $44.7(13.7)$ & $37.6(10.9)$ & $49.05(11.9)$ & $47.5(16.2)$ \\
\hline Female n (\%) & $50(83.3)$ & $18(81.8)$ & $18(81.8)$ & $14(87.5)$ \\
\hline TJC Median (IQR) & $4(12)$ & $0.5(4)$ & $5(13)$ & $10(11)$ \\
\hline SJC Median (IQR) & $3(8)$ & $0(3)$ & $4(12)$ & $7.5(9)$ \\
\hline DAS28-ESR Median (IQR) & & & $0.64(0.75)$ & $1.5(9)$ \\
\hline HAQ Median (IQR) & & & $18.07(16.8)$ & $23.8(15.5)$ \\
\hline CDAI MSedian (IQR) & & & $15(71.4)$ & $14(93.3)$ \\
\hline Married n (\%) & $41(68.3)$ & $12(54.5)$ & $10(45.5)$ & $4(25)$ \\
\hline Smoker n (\%) & $19(31.7)$ & $2(22.7)$ & & \\
\hline
\end{tabular}




\begin{tabular}{|l|c|c|c|c|}
\hline Morning Stiffness n (\%) & $31(51.7)$ & $4(18.2)$ & $16(72.7)$ & $11(68.8)$ \\
\hline RF IgM Positivity n (\%) & $46(76.7)$ & $9(40.9)$ & $12(70.6)$ & $4(25)$ \\
\hline ACPA Positivity $\mathrm{n}(\%)$ & $25(41.7)$ & $19(86.4)$ & $20(90.9)$ & $7(43.8)$ \\
\hline
\end{tabular}

Note: First degree relatives, FDR; Early Rheumatoid Arthritis, ERA; Late Rheumatoid Arthritis, LRA; Tender Joint Count, TJC; Swollen Joint Count, SJC; Disease Activity Score - Erythrocyte Sedimentation Rate, DAS28-ESR; Health Activity Questionnaire, HAQ; Clinical Disease Activity Index, CDAl; Rheumatoid Factor, RF; Anti citrullinated peptides antibodies, ACPA.

\begin{tabular}{|c|c|c|c|}
\hline \multicolumn{1}{|l|}{ Table 2: RAMRIS score divided by group. } \\
\hline & FDR & ERA & LRA \\
\hline Synovitis Score, Median(IQR) & $3.5(1.5)$ & $7(4)$ & $6.5(6.5)$ \\
\hline Erosion Score, Median(IQR) & $2(3.5)$ & $3.5(4)$ & $2.5(4.75)$ \\
\hline BME Score, Median(IQR) & $0(1.25)$ & $0(0.25)$ & $0(0.75)$ \\
\hline Total RAMRIS Score, Median(IQR) & $6(4.25)$ & $10(5)$ & $10(8.75)$ \\
\hline $\begin{array}{l}\text { Note: Rheumatoid Arthritis Magnetic Resonance Imaging Score, RAMRIS; First degree relatives, FDR; Early Rheumatoid Arthritis, ERA; Late } \\
\text { Rheumatoid Arthritis, LRA; Bone Marrow Edema, BME. }\end{array}$
\end{tabular}

\section{Discussion}

The overall performance of the AST to detect any synovitis in MCP joints was 0.575 in patients with CSA in order to establish the presence of synovitis. In a previous trial for diagnostic accuracy of the clinical positive squeeze test is associated with local joint inflammation but the sensitivity is low, indicating a high percentage of swollen joints with a negative squeeze test, and then poor diagnostic performance. As long as the global performance of AST to detect MRI abnormalities had no separation capability, we did not test the reported force previously, nor new force cutoffs. We tried to address the clinical variability and balance it with the AST compressor but the diagnostic performance is low nor predictive of MRI-detected abnormalities in MCP's. When the test is used on its own, it is insufficient to detect arthritis by MRI. Despite previous clinical results, besides ours, the squeeze test is commonly used in daily practice, even though the lack of evidence on it. It is possible that the additive prognostic value of clinical items (i.e. morning stiffness, first degree relative with RA, symptoms less than 1 year) that patients with high pretest probability to develop RA, confers the ST predictive performance on clinical grounds. Also need to consider that MRI is a very sensitive imaging method, contrasted with a non-specific test alone, could explain the results. It is important to evaluate the prognostic value of the AST not only in transversal and a sole clinical maneuver but as a reference tool with clinical characteristics and combined maneuvers (i.e. difficulty making a fist).

Other automated methods to detectsynovitis had been evaluated. Automated methods are referred to as techniques that require a small amount of effort and control from the performer. Werner et al. [14] evaluated 252 patients with arthritis and allied conditions and 12 controls ( 6 healthy individual and 6 with hand arthralgia) with indocyanine green (ICG)-enhanced fluorescence optical imaging (FOI), based on the concordance to histologically proven synovitis [14]. Using MRI as a reference, FOI had a sensitivity of $76 \%$ and a specificity of $54 \%$. As well in our study the synovitis gold standard was MRI, they used in 67 RA patients (52 female), with a mean of 58 years old (SD 8.3). The mean DAS28 was 3.8 (SD 1.0) and a mean RAMRIS synovitis score of 7 (SD 6). A $4.645 \mathrm{~kg}$ force of the AST had a $67 \%$ sensitivity and a $50 \%$ specificity, similar to FOI, our patients were younger and had more clinical activity. The main difference is that they used other arthritides in their evaluation; as long as the RAMRIS was developed to evaluate RA only, we do not use other kinds of patients. The different forces used in our AST do not need evaluation of an intra reader agreement as with FOI. Besselink et al. [15] evaluated 46 RA patients with no controls with optical spectral transmission (OST) model to measure joint inflammation, based on the correlation between joint inflammation, angiogenesis and light transmission through tissue [15].

Using ultrasound as a reference, OST had in MCP 1-5 joints a 0.88 AUC under ROC $(95 \%$ CI $0.84,0.92)$. With the optimal cut-off of 0.47 for all joints yielded a sensitivity of $60 \%$ and $89 \%$ specificity. The mean age of their patients was 60 (SD 13) and a mean DAS28ESR of 3.9 (SD 1.20). The presence of osteophytes or a soft tissue lesion that interferes with light transmission had to be taken in count to misinterpretation. Their population was older than ours, and the use of ultrasound as a reference made a difference to our findings. Ultrasound had been used as a reliable method to detect synovitis; recently Mueller et.al. reported the use of an automated ultrasound system in 19 RA and psoriatic arthritis patients with MCP joint inflammation [16]. Using MRI as a reference they reported an $83.5 \%$ sensitivity and a $44 \%$ specificity of automated ultrasound. There were $15 / 19$ women with a mean age of 50 years and a DAS28 of 4.5. The operator dependent issue was not reflected in this study, as long the manual ultrasound reflects similar performance.

Finally, Diekhoff et al. [17] used ultra-low-dose computed tomography (ULD-CT) in 36 RA patients. Using MRI as a reference ULD-CT had $69 \%$ sensitivity on the patient level and $65 \%$ on the joint level with $87 \%$ specificity. There were 26 women with a mean age of 60 years old. The mean synovitis RAMRIS score was 6.8 (SD 5.5) [17]. Even though CT was not as sensitive as MRI, 
the study reported more patient comfort compared with MRI. The Squeeze test (ST) is commonly used in daily practice, even though the lack of evidence on it; we demonstrated the great variability in their performance among rheumatologists. The ST had demonstrated more than $80 \%$ specificity to detect MCP arthritis [9]. Many primary care clinicians then advocate referral decisions on objective basis -autoantibodies positivity or a positive clinical maneuver. This phenomenon is more evident where there is scarcity of rheumatologists, and the referral policies on health systems are restricted. On the other hand, rheumatologists may rely on RA diagnosis in clinical grounds without serologic evidence, but may have the problem of subjectivity [18]. In conclusion, the AST does not have a great sensitivity or specificity to establish MCP joint inflammation. It does not show in an efficient way the synovitis inflammation changes seen on the MRI. Despite the fact the results found, research that involves automated devices should persist due to the importance of early identifying patients with high risk of developing RA.

\section{Conclusion}

The application of AST does not identify in an efficient way the synovitis seen on MRI.

\section{Acknowledgment}

Ivan de Jesús Hernández-Galarza, Luis Iván Lozano-Platas.

\section{Conflict of Interest Declaration}

None. All authors declare to have no conflicts of interest.

\section{Disclaimers}

None

\section{Source of Support}

The project has received funding for $\$ 300,000.00$ MXP from Dirección General de Planeación y Proyectos Estratégicos en el Programa para el Desarrollo Profesional Docente para el Tipo Superior (PRODEP) de la Universidad Autónoma de Nuevo León. Cuerpos Académicos (CA) number 136.

\section{References}

1. Botti C, Micillo A, Ricci G, Russo A, Denisco A, etal. (2018) Characterization of respiratory infection viruses in hospitalized children from Naples province in Southern Italy. Experimental and Therapeutic Medicine 15: 4805-4809.

2. Su W, Wu X, Geng X, Zhao X, Liu Q, et al. (2019) The short-term effects of air pollutants on influenza-like illness in Jinan, China. BMC Public Health 19:1319.

3. Lewis D (2020) Is the corona virus airborne? Experts can't agree. Nature 580: 175.

4. McGlone C (2020) Is the coronavirus hitching a ride on air pollution particles?

5. Offord C (2020) How COVID-19 is Spread. The Scientist.

6. Heymann DL, Shindo N (2020) Covid-19: What is next for public health. The Lancet 395(10224): 542-545.
7. Sohrabi C, Alsafi Z, O'Neill N, Khan M, Kerwan A, et al. (2020) World Health Organization declares global emergency: A review of the 2019 novel coronavirus (COVID-19). International Journal of Surgery 76: 7176.

8. NYT (2020) The New York Times Accessed.

9. Caiazzo F, Ashok A, Waitz IA, Yim SHL, Barrett SRH (2013) Air pollution and early deaths in the United States. Part I: Quantifying the impact of major sectors in 2005. Atmospheric Environment 79: 198-208

10. WHO (2014) 7 million premature deaths annually linked to air pollution, World Health Organization.

11. Kim D, Chen Z, Zhou LF, Huang SX (2018) Air pollutants and early origins of respiratory diseases. Chronic diseases and translational medicine 4(2): 75-94.

12. Xing YF, Xu YH, Shi MH, Lian YX (2016) The impact of $\mathrm{PM}_{25}$ on the human respiratory system. Journal of thoracic disease. 8(1): E69-E74.

13. Sioutas C, Delfino RJ, Singh M (2005) Exposure Assessment for Atmospheric Ultrafine Particles (UFPs) and Implications in Epidemiologic Research. Environmental Health Perspectives 113(8): 947-955.

14. Ostro B, Broadwin R, Green S, Feng WY, Lipsett M (2006) Fine particulate air pollution and mortality in nine California counties: results from CALFINE. Environ Health Perspect 114: 29-33.

15. Kim K, Kabir E, Kabir S (2015) A review on the human health impact of airborne particulate matter. Environment International 74: 136-143.

16. Silva DR, Viana VP, Müller AM, Livi FP, Dalcin PT (2014) Respiratory viral infections and effects of meteorological parameters and air pollution in adults with respiratory symptoms admitted to the emergency room. Influenza Other Respir Viruses 8: 42-52.

17. ALA (2020) Bacteria and Viruses. American Lung Association.

18. Pan M, Lednicky JA, Wu CY (2016) Collection, particle sizing and detection of airborne viruses. Journal of Applied Microbiology 127(6): 1596-1611.

19. Feng C, Li J, Sun W, Zhang Y, Wang Q (2016) Impact of ambient fine particulate matter $\left(\mathrm{PM}_{25}\right)$ exposure on the risk of influenza-like-illness: a time-series analysis in Beijing, China. Environmental Health 16(15): 17.

20. Donaldson K, Stone V, Clouter A, Renwick L, MacNee W (2001) Ultrafine particles. Occupational and Environmental Medicine 58: 211-216.

21. CDC (2019) Coronavirus Disease 2019 COVID-19. Public Health Recommendations for Community-Related Exposure. Center for Disease Control and Prevention.

22. Groulx N, Urch B, Duchaine C, Mubareka S, Scott JA (2018) The Pollution Particulate Concentrator (PoPCon): A platform to investigate the effects of particulate air pollutantson viral infectivity. The Science of the Total Environment 628-629: 1101-1107.

23. Gralton J, Tovey ER, McLaws ML, Rawlinson WD (2013) Respiratory virus RNA is detectable in airborne and droplet particles. Journal of Medical Virology 85(12): 2151-2159.

24. Xu Z, Hu W, Williams G, Clements AC, Kan H, et al. (2013) Air pollution, temperature and pediatric influenza in Brisbane, Australia. Environ Int 59: 384-388.

25. Huang L, Zhoub L, Chena J, Chena K, LiuY, et al. (2016) Acute effects of air pollution on influenza-like illness in Nanjing, China: A population-based study. Chemosphere 147: 180-187.

26. Liu XX, Li Y, Qin G, Zhu Y, Li X, et al. (2019) Effects of air pollutants on occurrences of influenza-like illness and laboratory-confirmed influenza in Hefei, China. Int J Biometeorol 63(1):51-60.

27. Croft DP, Zhang W, Lin S, Thurston SW, Hopke PK, et al. (2019) The Association between Respiratory Infection and Air Pollution in the 
Setting of Air Quality Policy and Economic Change. Annals of the American Thoracic Society 16(3): 321-330.

28. Wu X, Nethery RC, Sabath BM, Braun D, Dominici F (2020) Exposure to air pollution and COVID-19 mortality in the United States med Rxiv.

29. Hornyak T (2020) Air pollution can worsen the death rate from COVID-19. Eos 101.

30. Ghio AJ, Soukup JM, Madden MC (2018) The toxicology of air pollution predicts its epidemiology. Inhalation Toxicology 30(9-10): 327-334.

31. Schiermeier Q (2020) Why pollution is plummeting in some cities - but not others.

32. Becker S, Soukup JM (1999) Exposure to urban air particulates alters the macrophage-mediated inflammatory response to respiratory viral infection. J Toxicol Environ Health A 57(7): 445-457.

33. Karr CJ,Rudra CB, Miller KA, Gould TR, Larson T, et al. (2009) Infant exposure to fine particulate matter and traffic and risk of hospitalization for RSV bronchiolitis in a region with lower ambient air pollution. Environ Res 109(3): 321-327.

34. Khilnani GC, Tiwari P (2018) Air pollution in India and related adverse respiratory health effects: past, present, and future directions. CurrOpinPulm Med 24(2): 108-116.

35. Landrigan PJ (2017) Air pollution and health. Lancet Public Health 2(1): e4-e5.
36. Li Y, Leung GM, Tang JW, Yang X, Chao CY, et al. (2007) Role of Ventilation in Airborne Transmission of Infectious Agents in the Built Environment - A Multidisciplinary Systematic Review. Indoor Air 17(1): 2-18.

37. National Research Council (NRC) (2006) Green Schools: Attributes for Health and Learning. Committee to Review and Assess the Health and Productivity Benefits of Green Schools, National Academies Press, USA.

38. NJDEP (n.d.) Bureau of Air Quality Planning. Retrieved April 28, 2013, from NJDEP.

39. Paulin L, Hansel N (2016) Particulate air pollution and impaired lungfunction. F1000 Research 5 [7108.1].

40. RWJF (2012) How does transportation impact health? Robert Wood Johnson Foundation. Health Policy Snapshot Series.

41. UOT (2018) Airborne interactions between viruses and PM2.5 as an unexplored modifier of viral viability. University of Toronto.

42. Vandini S, Corvaglia L, Alessandroni R, Aquilano G, Marsico C, et al. (2013) Respiratory syncytial virus infection in infants and correlation with meteorological factors and air pollutants. Italian journal of paediatrics 39(1): 1 .

43. WHO (2014) Infection Prevention and Control of Epidemic-and Pandemic-Prone Acute Respiratory Infections in Health Care, Geneva, Switzerland: World Health Organization. 